\title{
Beyond Plurality: Truth-Bias in Binary Scoring Rules
}

\author{
Svetlana Obraztsova ${ }^{1}$, Omer Lev $^{2}$, Evangelos Markakis ${ }^{3}$, Zinovi Rabinovich ${ }^{4}$, and \\ Jeffrey S. Rosenschein ${ }^{2}$ \\ ${ }^{1}$ Tel Aviv University \\ ${ }^{2}$ Hebrew University of Jerusalem \\ svet lana.obraztsovalgmail.com \\ ${ }^{3}$ Athens University of Economics and Business \\ markakis@gmail.com \\ \{omerl, jeff\}@cs.huji.ac.il \\ ${ }^{4}$ Mobileye Vision Technologies Ltd. \\ zrezinovi.net
}

\begin{abstract}
It is well known that standard game-theoretic approaches to voting mechanisms lead to a multitude of Nash Equilibria (NE), many of which are counter-intuitive. We focus on truth-biased voters, a model recently proposed to avoid such issues. The model introduces an incentive for voters to be truthful when their vote is not pivotal. This is a powerful refinement, and recent simulations reveal that the surviving equilibria tend to have desirable properties.

However, truth-bias has been studied only within the context of plurality, which is an extreme example of $k$-approval rules with $k=1$. We undertake an equilibrium analysis of the complete range of $k$-approval. Our analysis begins with the veto rule, the other extreme point of $k$-approval, where each ballot approves all candidates but one. We identify several crucial properties of pure NE for truth-biased veto. These properties show a clear distinction from the setting of truth-biased plurality. We proceed by establishing that deciding on the existence of NE in truth-biased veto is an NP-hard problem. We also characterise a tight (in a certain sense) subclass of instances for which the existence of a NE can be decided in poly-time. Finally, we study analogous questions for general $k$-approval rules.
\end{abstract}

\section{Introduction}

Voting mechanisms are processes by which preferences can be aggregated, and collective decisions can be made, in various multi-agent contexts. Under most voting rules, potentially beneficial strategic behavior is essentially inherent, as the GibbardSatterthwaite theorem famously states $[7,16]$. Hence, under mild assumptions, voters may have incentives to misreport their preferences. Given this negative result, a natural approach, initiated by Farquharson [5], is to undertake a game-theoretic analysis of voting, viewing voters as strategic agents, and examining the set of Nash equilibria of the underlying game.

However, most voting games contain an enormous amount of Nash equilibria, with even small games reaching hundreds of thousands of equilibria. Furthermore, many of the equilibria are votes which will not occur in the real world (e.g., for most voting rules, if all voters rank the same candidate last, the case where all voters vote for this least favorite option is a Nash equilibrium). Therefore, there has been very little analysis regarding the structure of the different equilibria — such an analysis would not be 
informative about the voting procedure and its quality. Moreover, without an understanding of strategic effects on voting, the ability to compare voting rules and choose an appropriate one for each setting is very limited.

In the past few years, several ideas have been raised regarding sensible limitations on the structure of games or equilibria, in order to provide a better game-theoretic analysis of voting scenarios. One of the most popular ideas, raised both in the social choice literature [3] and in the computer-science literature [17,11] is truth-bias. Truth bias means that in scenarios in which the voter has no way to manipulate via an insincere vote, in order to improve the result, the voter prefers to stick to its actual preferences and vote truthfully. Such a behavior indeed eliminates many nonsensical equilibria, and generally reduces the number of equilibria in voting games [17].

Contribution: While truth-bias has been analyzed and explored for plurality, it has yet to be extended to other voting rules, and this paper advances our understanding of the effect of truth-bias on other rules. It is not clear a priori that the same structural properties that were identified for plurality under truth bias will hold for different rules (in fact, as we show, they generally do not). Consequently, we embark on handling the voting rules most closely related to plurality - the veto rule and the more general family of $k$-approval voting. These rules, together with plurality, are those that contain only two possible values that voters can give to candidates (approve or disapprove, veto or not veto). We focus on analyzing the pure Nash equilibria under truth bias and we examine and identify a variety of their key properties. One of the crucial features in these schemes is the performance of winners and runner-up candidates at non-truthful equilibria, i.e., their score compared against the score of the winner under truthful voting. These scores can vary in different ways, resulting in different equilibrium configurations in each rule. Such comparisons with the existing work on plurality $[17,14]$ are summarized in Table 2 of Section 4.

We begin by focusing on the veto voting rule, where each voter chooses a single candidate from whom to withhold a point. We first characterize its truth-biased equilibria. We then further our results to describe an algorithm that-using max-flow considerations-is able to discern, under certain conditions, whether there exists an equilibrium or not. ${ }^{1}$ Moreover, we are able to show that our result is tight to an extent, as removing even one of the identified conditions results in an NP-complete problem.

Following that, we consider the $k$-approval rule, where we examine the same set of questions; we demonstrate that the landscape is more complex for $k$-approval. Our results move us towards a better understanding of the effects of truth bias on each of these voting rules. As we show, we obtain quite different properties under each rule, and we elaborate more on this in Section 5.

\subsection{Related Work}

There have been many modeling approaches towards eliminating the multitude of Nash equilibria in voting games. Some are based on introducing uncertainty, either regarding the support of each candidate [13], or about the reliability of counting procedures [12]. Other research suggests changing the temporal structure of the game; for example, Xia

\footnotetext{
${ }^{1}$ Unlike regular, non-truth-biased voting games, with truth-bias there are scenarios where there is no Nash equilibrium at all. This has also been shown for plurality.
} 
and Conitzer [18] and Desmedt and Elkind [2] consider the case where agents vote publicly and one-at-a-time, and study subgame-perfect equilibria of these extensiveform games. A different approach is the notion of lazy voting [2], where the utility function is changed so that non-pivotal voters have a slight preference to abstain.

Another way to refine the equilibria set is to stick to the basic game theoretic models, but study NEs that are reachable by iterative voting procedures. The iterative voting model was introduced by Meir et al. [11] and later expanded by Lev and Rosenschein [10] and Branzei et al. [1]; this work followed the research into iterative and dynamic mechanisms, chiefly summarised by Laffont [8]. Interestingly, Branzei et al. [1] show that under plurality, the reachable equilibria of this process are of relatively "good" quality.

We focus on a different model than the approaches above for refining the set of equilibria in a voting game, that of truth bias. The notion of adding a truth bias has been introduced into multiple game theoretic models before, albeit for specialised cases. E.g. by Dutta and Sen [4] for a mechanism design model, and by Laslier and Weibull [9] and by Dutta and Laslier [3] for limited voting scenarios. A more robust model was suggested by Thompson et al. [17], which introduced the general framework, and contained various empirical results for the plurality rule in truth-biased games. The theoretical side of that work was enhanced by Obraztsova et al. [14]. More recent work has also attempted to relate this line of work to iterative voting [15], but this again is solely with respect to plurality.

\section{Definitions and Notation}

We consider a set of $m$ candidates $C=\left\{c_{1}, \ldots, c_{m}\right\}$ and a set of $n$ voters $V=$ $\{1, \ldots, n\}$. Each voter $i$ has a preference order (i.e., a ranking) over $C$, which we denote by $a_{i}$. For notational convenience in comparing candidates, we will often use $\succ_{i}$ instead of $a_{i}$. When $c_{k} \succ_{i} c_{j}$ for some $c_{k}, c_{j} \in C$, we say that voter $i$ prefers $c_{k}$ to $c_{j}$.

At an election, each voter submits a preference order $b_{i}$, which does not necessarily coincide with $a_{i}$. We refer to $b_{i}$ as the vote or ballot of voter $i$. The vector of submitted ballots $\mathbf{b}=\left(b_{1}, \ldots, b_{n}\right)$ is called a preference profile. At a profile $\mathbf{b}$, voter $i$ has voted truthfully if $b_{i}=a_{i}$. Any other vote from $i$ will be referred to as a non-truthful vote. Similarly the vector $\mathbf{a}=\left(a_{1}, \ldots, a_{n}\right)$ is the truthful preference profile, whereas any other profile is a non-truthful one.

A voting rule $\mathcal{F}$ is a mapping that, given a preference profile $\mathbf{b}$ over $C$, outputs a candidate $c \in C$, the election's winner; we write $c=\mathcal{F}(\mathbf{b})$. In this paper we will consider the veto rule, as well as the more general family of $k$-approval rules. This is a family of voting rules that let each voter divide candidates into two groups: the first one is the approved group, of size $k$ (the top $k$ candidates ranked by the voter), and each candidate within the group gets a point from this voter. The rest of the candidates compose the second group, and receive no points. The ranking within each group in the submitted ballot does not matter. The candidate with the most points is the winner of the election, and we resolve ties using lexicographic tie-breaking. The veto rule, in which all candidates but one are given a point is an extreme member of this family. The other extreme is plurality, for which many of the issues dealt with in this paper have been resolved by Obraztsova et al. [14]. We denote by $\operatorname{sc}(c, \mathbf{b})$ the score of candidate $c \in C$ in a voting profile $\mathbf{b}$. 
In this work, we view elections as a non-cooperative game, in which a utility function $u_{i}$ is associated with every voter $i$, that is consistent with its true preference order. That is, we require that $u_{i}\left(c_{k}\right) \neq u_{i}\left(c_{j}\right)$ for every $i \in V, c_{j}, c_{k} \in C$, and also that $u_{i}\left(c_{k}\right)>u_{i}\left(c_{j}\right)$, if and only if $c_{k} \succ_{i} c_{j}$. We let $p_{i}\left(a_{i}, \mathbf{b}, \mathcal{F}\right)$ denote the utility of voter $i$, when $a_{i}$ is its true preference ranking, $\mathbf{b}$ is the submitted profile by all voters, and $\mathcal{F}$ is the voting rule under consideration, and the utility function of voter $i$ can be constructed from its $p_{i}$ function. In the common model of election, this means $p_{i}\left(a_{i}, \mathbf{b}, \mathcal{F}\right)=u_{i}(\mathcal{F}(\mathbf{b}))$. A Nash equilibrium in these games is a profile $\mathbf{b}^{N E}$, where no voter has an incentive to unilaterally deviate, i.e., for every $i$ and for every vote $b_{i}^{\prime}$, we have $p_{i}\left(a_{i}, \mathbf{b}^{N E}, \mathcal{F}\right) \geq p_{i}\left(a_{i},\left(b_{i}^{\prime}, \mathbf{b}_{-i}^{N E}\right), \mathcal{F}\right)$, where $\mathbf{b}_{-i}^{N E}$ is the vector $\mathbf{b}^{N E}$ without player $i$ 's vote.

However, such a model is known to result in multiple equilibria, including nonsensical ones. Assume, for example, that all voters have the same preferences, which coincide with the tie-breaking order; then the profile where all of them veto their favourite candidate is an equilibrium. We can construct many other undesirable equilibria. Hence, we instead focus on the more promising truth-biased model [17]. In this model, we suppose that voters have a slight preference for voting truthfully when they cannot unilaterally affect the outcome of the election. This bias is captured by inserting a small extra payoff when the voter votes truthfully. This extra gain is small enough so that voters may still prefer to be non-truthful in cases where they can affect the outcome. If $\mathbf{a}$ is the real profile, $\mathbf{b}$ is the submitted one, and $\epsilon$ a very small value, the payoff function of voter $i$ is given by:

$$
p_{i}\left(a_{i}, \mathbf{b}, \mathcal{F}\right)= \begin{cases}u_{i}(\mathcal{F}(\mathbf{b})), & \text { if } a_{i} \neq b_{i}, \\ u_{i}(\mathcal{F}(\mathbf{b}))+\epsilon, & \text { if } a_{i}=b_{i} .\end{cases}
$$

As already described in Section 1.1, this model has recently gained popularity, since it achieves a significant refinement of the set of Nash equilibria, and it has been analyzed in previous work under the plurality voting rule.

Now, the following two equilibrium-related problem classes are of interest. The first deals with determining the existence of equilibria in such a voting game, whereas the second asks about the existence of equilibria with a given candidate as a winner.

Definition $1(\exists N E)$. An instance of the $\exists N E$ problem is determined by a preference profile a, and will be denoted by $\exists N E(\mathbf{a})$. The profile a indicates the true preferences of the voters. Given a, $\exists N E(\mathbf{a})$ is a "yes" instance $\Longleftrightarrow$ the corresponding election game, with truth-biased voters, admits at least one Nash equilibrium.

Definition 2 (WinNE). An instance of the WinNE problem is determined by a preference profile $\mathbf{a}$, and a candidate $w \in C$, denoted by $\operatorname{Win} N E(w, \mathbf{a})$. It is a "yes" instance $\Longleftrightarrow$ the corresponding election game, with truth-biased voters, admits at least one Nash equilibrium with $w$ as the winner.

\section{Truth-bias under the Veto Voting Rule}

In this section we provide an analysis of pure Nash equilibria, under the assumption that voters are truth-biased and use the veto voting rule. Our main focus is on how nontruthful equilibrium profiles $\mathbf{b} \neq \mathbf{a}$ can arise. Whether the truthful profile itself is a Nash equilibrium can be decided easily. Some proofs are omitted due to space limitations. 


\subsection{Properties of Nash Equilibria under Truth-bias}

We begin by defining a class of candidates, which will become useful further on:

Definition 3. In a profile $\mathbf{b}$, where the winner is $\mathcal{F}(\mathbf{b})$, a runner-up candidate is a candidate $c \in C$, for which one of the following conditions hold:

- $\operatorname{sc}(c, \mathbf{b})=\operatorname{sc}(\mathcal{F}(\mathbf{b}), \mathbf{b})$, and $\mathcal{F}(\mathbf{b}) \succ c$ in the tie-breaking rule,

- $\operatorname{sc}(c, \mathbf{b})=\operatorname{sc}(\mathcal{F}(\mathbf{b}), \mathbf{b})-1$, and $c \succ \mathcal{F}(\mathbf{b})$ in the tie-breaking rule.

Essentially, a runner-up candidate is a candidate that could become a winner by gaining one extra point. We will denote the set of runner-up candidates that satisfy the first (respectively, second) condition of the definition above by $\mathbf{R}_{1}$ (respectively, $\mathbf{R}_{2}$ ). Similarly to the analysis of the truth-biased plurality rule in [14], we define here a notion of a threshold candidate. Our definition, however, is different, and tailored to our veto rule analysis. Intuitively, the threshold candidate is the candidate that would become a winner if the current winner, $\mathcal{F}(\mathbf{b})$, lost a point, as can be seen from the definition below.

Definition 4. Given a voting profile $\mathbf{b}$, a threshold candidate $c$ is a runner-up candidate for which one of the following holds:

- $c$ is the maximal element of $\mathbf{R}_{1}$ w.r.t. the tie-breaking order, if $\mathbf{R}_{1} \neq \emptyset$,

- $c$ is the maximal element of $\mathbf{R}_{2}$ w.r.t. the tie-breaking order, if $\mathbf{R}_{1}=\emptyset$.

The next important lemma considers the score of a winner at an equilibrium.

Lemma 1. Let $\mathbf{b}^{N E} \neq \mathbf{a}$ be a non-truthful Nash equilibrium, with $w=\mathcal{F}\left(\mathbf{b}^{N E}\right)$. The score of the winner, $w$, in $\mathbf{b}^{N E}$, is the same as its score at the truthful profile, i.e., $\operatorname{sc}(w, \mathbf{a})=\operatorname{sc}\left(w, \mathbf{b}^{N E}\right)$.

Proof. Suppose $\operatorname{sc}\left(w, \mathbf{b}^{N E}\right)>\operatorname{sc}(w, \mathbf{a})$. This means that there is a voter $i \in V$, that gives $w$ a point which it would not give under the truthful profile. That is, it is giving a point to its least-favorite candidate. Such a voter can certainly gain by switching back to its truthful vote. In that case, either a new winner emerges, which would be above $w$ in the preference ranking of $i$, or $w$ remains the winner, but $i$ gets a higher utility by $\epsilon$, due to voting truthfully.

Now suppose $\operatorname{sc}\left(w, \mathbf{b}^{N E}\right)<\operatorname{sc}(w, \mathbf{a})$, i.e., a voter $i \in V$ is vetoing $w$ in $\mathbf{b}^{N E}$, but not in the truthful a. Yet, returning to its truthful vote, $a_{i}, w$ will still remain the winner, and this will increase player $i$ 's utility by $\epsilon$, due to the truth-bias.

In fact, we can further show that not only the winner's score does not change at a non-truthful equilibrium, but the set of voters which support the winner are the same as in the truthful profile. Hence, we obtain the following:

Corollary 1. Let $\mathbf{b}^{N E} \neq \mathbf{a}$ be a non-truthful Nash equilibrium, with $w=\mathcal{F}\left(\mathbf{b}^{N E}\right)$. The set of voters that veto $w$ in $\mathbf{a}$ is the same set that vetoes $w$ in $\mathbf{b}^{N E}$.

The next properties that we identify are simple to prove but crucial in understanding what equilibria look like under the veto rule. 
Lemma 2. For any non-truthful equilibrium profile $\mathbf{b}^{N E} \neq \mathbf{a}$, there always exists a threshold candidate in $\mathbf{b}^{N E}$.

Proof. It suffices to show that there always exist runner-up candidates; hence, there is a threshold runner-up as well. Let $\mathbf{b}^{N E} \neq \mathbf{a}$ be an equilibrium with $w=\mathcal{F}\left(\mathbf{b}^{N E}\right)$. Suppose we have a non-truthful equilibrium and that there is no runner-up candidate. Consider a voter $i$ that voted non-truthfully. By Corollary 1, the non-truthful voters in $\mathbf{b}^{N E}$ do not veto $w$ (and they do not veto $w$ in a either). Hence $i$ has vetoed some other candidate. By switching back to its truthful vote, the outcome is not going to change, since there is no runner-up candidate and since $w$ is not going to lose any points. Hence $i$ is better off by $\epsilon$ to vote truthfully, a contradiction. Thus there are always runner-up candidates at a non-truthful equilibrium.

Observation 1 All voters that do not veto the winner or the runner-ups in an equilibrium profile prefer the winner over the threshold candidate (otherwise, they could just veto the winner and make the threshold candidate win).

Example 1. There are cases where the threshold candidate in an equilibrium may have fewer points than in the truthful state (note that this is not true for plurality, as shown in Lemma 2 of Obraztsova et al. [14]); we show it here with an example of 4 candidates. Suppose the tie-breaking rule is $c \succ b \succ d \succ w$, and the truthful profile is:

- 3 voters with ranking: $w \succ b \succ c \succ d \quad \bullet 2$ voters with ranking: $w \succ d \succ c \succ b$

- 1 voter with ranking: $w \succ b \succ d \succ c \quad \bullet 1$ voter with ranking: $b \succ c \succ d \succ w$

Then $c$ is the winner of the truthful profile. In turn, in the following profile, $w$ is the winner and the threshold candidate is $c$, which has fewer points than in the truthful state. Notice that the profile is an equilibrium, and just one voter has moved from the first group to the 3rd one:

- 2 voters with: $w \succ b \succ c \succ d \quad \bullet 2$ voters with: $w \succ d \succ c \succ b$

- 2 voter with: $w \succ b \succ d \succ c \quad \bullet 1$ voter with: $b \succ c \succ d \succ w$

Finally, to facilitate our discussion in the next subsections, we define the concept of "voting against" a candidate, and a simple companion lemma.

Definition 5. We will say that a voter $j$ votes against candidate $c_{i}$ in a profile $\mathbf{b}$, if $b_{j} \neq a_{j}$ and $c_{i}$ is vetoed in $b_{j}$.

Lemma 3. In every non-truthful NE, all non-truthful voters vote against some runnerup candidate in the NE (not necessarily the same one).

\subsection{Existence of Nash Equilibria}

Having identified the properties above, we are now ready to prove our first set of results regarding the complexity of the problems WinNE and $\exists N E$, as defined in Section 2. We start with the following negative result.

Theorem 1. Under the veto rule and with truth-biased voters the problem WinNE is NP-complete. 
Proof. While membership in NP is trivial, completeness requires several steps. We will construct a reduction from exact-cover by 3 -sets (X3C).

Definition 6. Exact cover by 3 sets (X3C) is a problem in which we have a set of $3 \mathrm{~m}$ elements $U=\left\{u_{1}, \ldots, u_{3 m}\right\}$ and a set of sets $S=\left\{S_{1}, \ldots, S_{n}\right\}$ such that for $1 \leq i \leq$ $n: S_{i} \subseteq U,\left|S_{i}\right|=3$. We wish to know if there is a set $T \subseteq S$ such that $|T|=m$ and $\cup_{S \in T} S=U$ (NP-completness shown in [6])

Taking an $\mathrm{X} 3 \mathrm{C}$ instance, we construct an instance of our problem. Our candidates will be the members of $S$ and $U$, to which we add two new candidates $w$ and $t$. To construct our voters, we introduce some markings to aid us: $S_{i}$ 's elements are $\left\{u_{i_{1}}, u_{i_{2}}, u_{i_{3}}\right\}$, and we denote by $\mathcal{S}$ the members of $S$ ordered as usual $-S_{1} \succ S_{2} \succ$ $\ldots \succ S_{n}$; similarly we use $\mathcal{U}$ for the ordering of $U . \overline{\mathcal{S}}$ marks the opposite direction $S_{n} \succ S_{n-1} \succ \ldots \succ S_{1}$, and ditto for $\overline{\mathcal{U}}$. Our tie-breaking rule is $w \succ t \succ \mathcal{S} \succ \mathcal{U}$. We now describe the set of voters, which consists of the two blocks of voters described in Table 1, along with 3 more blocks described below:

- Block 3: For every $u_{i} \in U$, we have:

- $m$ votes of the form: $\mathcal{U} \backslash\left\{u_{i}\right\} \succ \mathcal{S} \succ w \succ t \succ u_{i}$;

- $n-2 m-1$ votes of the form: $\mathcal{\mathcal { S }} \succ \overline{\mathcal{U}} \backslash\left\{u_{i}\right\} \succ w \succ t \succ u_{i}$.

- Block 4: For every $S_{i} \in S$, we have:

- $m$ votes of the form: $\mathcal{S} \backslash\left\{S_{i}\right\} \succ \mathcal{U} \succ w \succ t \succ S_{i}$;

- $n-2 m-1$ votes of the form: $\overline{\mathcal{U}} \succ \overline{\mathcal{S}} \backslash\left\{S_{i}\right\} \succ w \succ t \succ S_{i}$.

- Block 5: $n-m$ votes of the form: $t \succ \mathcal{S} \succ \mathcal{U} \succ w$.

Table 1. NP-Completeness proof profiles.

\begin{tabular}{|lll|}
\hline \multicolumn{3}{|c|}{ Block 1 } \\
\hline \hline $\mathcal{S} \backslash\left\{S_{1}\right\}, \overline{\mathcal{U}}$, & $\ldots \mathcal{S} \backslash\left\{S_{n}\right\}$ \\
$\mathcal{U}$, & $\overline{\mathcal{S}} \backslash\left\{S_{2}\right\}, \ldots \mathcal{U}$ \\
$w$, & $w$, & $\ldots w$ \\
$S_{1}$, & $S_{2}$, & $\ldots S_{n}$ \\
$t$, & $t$ & $\ldots t$ \\
\hline
\end{tabular}

\begin{tabular}{|c|c|c|c|}
\hline \multicolumn{4}{|c|}{ Block 2} \\
\hline \multicolumn{4}{|c|}{$\ldots \mathcal{U} \backslash\left\{u_{i_{1}}\right\}, \mathcal{U} \backslash\left\{u_{i_{2}}\right\}, \mathcal{U} \backslash\left\{u_{i_{3}}\right\}, \ldots$} \\
\hline$\ldots \mathcal{S} \backslash\{$ & $\mathcal{S} \backslash\{$ & $\mathcal{S} \backslash\{$ & \\
\hline$\ldots w$ & $w$ & $w$ & \\
\hline$\ldots t$, & $t$ & $t$ & $\cdots$ \\
\hline$\ldots u_{i_{1}}$, & $u_{i_{2}}$ & $u_{i_{3}}$ & $\cdots$ \\
\hline$\ldots S_{i}$ & $S_{i}$ & $S_{i}$ & \\
\hline
\end{tabular}

In the truthful profile, $w$ is not the winner ( $u_{1}$ is). We claim that there is an equilibrium in which $w$ is the winner if and only if there is a solution to the $\mathrm{X} 3 \mathrm{C}$ problem.

Lemma 4. Given the constructed truthful profile, if a NE profile $\mathbf{b}^{N E}$ exists with $w$ as a winner, $t$ is the threshold candidate in $\mathbf{b}^{N E}$.

Proof. Consider an equilibrium $\mathbf{b}^{N E}$, and let us assume the opposite: $t$ is not a threshold candidate. Consider the possibility that $S_{i}$ is the threshold candidate of $\mathbf{b}^{N E}$. Let us take a closer look at a voter from Block 4 of the form $\mathcal{S} \backslash\left\{S_{j}\right\} \succ \mathcal{U} \succ w \succ t \succ S_{j}$, for some $j \neq i$ so that $S_{j}$ is not a runner-up. In the equilibrium $\mathbf{b}^{N E}$, this voter can veto only either $S_{j}$ or $t$. Furthermore, since $t$ beats all candidates except $w$ in tie breaking, if $t$ is not the threshold candidate then it cannot be a runner-up candidate at all. As a result, the inspected voter cannot veto $t$, according to Lemma 3 . Thus, we have found a voter that votes truthfully in the NE profile $\mathbf{b}^{N E}$ and prefers $S_{i}$ to $w$, contradicting Observation 1. 
Consider now the case where all $S_{i}$ 's are runner-up candidates. In this case, each $S_{i}$ has gained two more points relatively to their respective scores in the truthful profile a. Only voters in Block 2 are capable of deviating to accomplish this (the reasoning in the paragraph above is why Block 4 votes will not deviate). In particular, for each $S_{i}$ there are at least two such voters that deviate to veto $u_{i_{l}}$ and $u_{i_{k}}$ for some $l \neq k \in\{1,2,3\}$. As a result there are at least 2 additional voters (in comparison to the truthful profile) that veto candidates from $\mathcal{U}$. The Pigeonhole Principle dictates that there is at least one $u_{i_{l}}$ that has received two more veto votes in comparison to the truthful profile. In addition, notice that there can be no voter in the NE profile $\mathbf{b}^{N E}$ that deviates in favor of $u_{i_{l}}$. As a result, $u_{i_{l}}$ cannot be a runner-up candidate, yet has voters that deviate to veto it, in contradiction to Lemma 3. Hence, it is impossible for all $S_{i}$ 's to be runner-up candidates simultaneously.

Similarly, we will obtain a contradiction for the case where we assume that $u_{i}$ is the threshold candidate, and, therefore, none of the $S_{i}$ 's are runner-ups.

We conclude that in a NE profile $\mathbf{b}^{N E}, t$ must be the threshold candidate and it will have $(n-m)$ points. Let us now proceed with the remainder of the proof of Theorem 1 .

If there is $T=\left\{S_{1}^{\prime}, \ldots, S_{m}^{\prime}\right\} \subseteq S$ which is a solution to the X3C problem, we have an equilibrium in which $w$ is the winner: the voters from Block 1 whose penultimate candidate is $S_{i}^{\prime} \in T$ will veto $S_{i}^{\prime}$. The voters in Block 2 who veto $S_{i}^{\prime} \in T$ instead veto their penultimate candidates $u_{i_{1 / 2} / 3}$. In such a situation all candidates are vetoed by $n-$ $m$ voters (apart from those in $S \backslash T$, which are vetoed by $n-m+2$ voters), and therefore $w$ is the winner. All voters are vetoing runner-ups which they prefer less than $w$ or $t$. Hence, changing their vote will make the candidate they currently veto the winner, and as they would rather have $w$ win, they do not change their vote. Furthermore, all voters from Blocks 1 through 4 that do not veto a runner-up candidate, can only deviate so that $t$ becomes a winner. Since they prefer $w$ to $t$, none of them will actually have an incentive to deviate. Finally, none of the voters in Block 5 can change the election outcome and will remain truthful.

Now, assume that there is no solution to the $\mathrm{X} 3 \mathrm{C}$ problem. At least $m$ voters from Block 1 will veto the $S_{i}$ 's (the only candidates less-preferred than $w$ ). However, in order for them not to revert to their truthful vote, those $S_{i}$ 's need to be runner-up candidates, so all votes in Block 2 who would truthfully veto those $S_{i}$ 's, need to veto their respective $u_{i}$ 's instead. In addition, those $u_{i}$ 's need to be runner-ups as well (or those Block 2 votes will revert to the truthful vote), and as they are ranked below $S$ in the tie-breaking rule, they need to have $m-n$ vetoes in order to be runner-ups. This means that each $u_{i}$ is vetoed only once in Block 2 . So we have $m$ (or more) $S_{i}$ 's containing exactly one copy of each $u_{i}$; i.e., we found an exact cover of $U$, contradicting the assumption that X3C has no solution.

The above proof also implies Corollary 2. Furthermore, its variant can also be used to prove a more general theorem (due to lack of space, the proof is not presented here).

Theorem 2. Consider the veto rule and truth-biased voters. Then the problem $\exists N E$ is NP-complete.

It is possible to further expand upon the result of Theorem 1. To this end, we identify two conditions that help us characterize the set of computationally hard instances. In 
particular, consider an instance $\operatorname{Win} N E(w, \mathbf{a})$, determined by a candidate $w \in C$ and a truthful profile $\mathbf{a}$. We consider the following conditions:

C1: Let $t \in C$ be the candidate right below $w$ in the tie-breaking order (i.e., the tiebreaking order is in the form $\cdots \succ w \succ t \succ \cdots)$. Then $\operatorname{sc}(t, \mathbf{a}) \geq \operatorname{sc}(w, \mathbf{a})$.

C2: Let $t$ be as in C1. Then, for every voter $i$ that does not veto $w$ in the truthful profile a, it holds that $w \succ_{i} t$.

Corollary 2. Under the veto voting rule, the problem WinNE is NP-complete, even for the family of instances that satisfy condition $C 2$ and do not satisfy condition C1.

In fact, together with Theorem 3, the picture becomes more clear regarding hardness results: violating either one of the conditions $\mathrm{C} 1, \mathrm{C} 2$ makes the problem WinNE hard.

Theorem 3. Under the veto voting rule, the problem WinNE is NP-complete, even for the family of instances where C1 holds but C2 does not.

Proof. As with Theorem 1, we will construct a reduction from X3C. We will use the same notation, where $S$ is the set of sets and $U$ is the set of elements in an instance of $\mathrm{X} 3 \mathrm{C}$, and convert the members of these sets into distinct candidates. However, unlike the previous proof, in addition to the candidates from $S$ and $U$, we will introduce four special candidates $w, t, p_{1}$ and $p_{2}$.

Based on the candidate set defined above, we will construct a set of voters and their truthful preference profile $\mathbf{a}$, so that a solution to $\operatorname{Win} N E(w, \mathbf{a})$ would entail a solution to the $\mathrm{X} 3 \mathrm{C}$ instance.

We will order the candidates to form the following tie-breaking preference order: $w \succ t \succ p_{1} \succ p_{2} \succ S \succ U$, where candidates from $S$ and $U$ appear in their natural lexicographic order.

We now construct a set of voters, grouped into five distinct blocks, according to their truthful preference profile. In each block we only explicitly describe the order of a few least-preferred candidates. All candidates that are not explicitly mentioned in a profile, appear in an arbitrary order, and are marked by ....

- Block 1: A set of $n$ voters, one for each candidate in $S$, with preference profile of the form $\cdots \succ t \succ w \succ S_{i} \succ p_{1}$;

- Block 2: A set of $n-m$ voters with preference profile of the form $\cdots \succ t \succ w \succ p_{2}$ and one additional voter with profile of the form $\cdots \succ w \succ t \succ p_{2}$;

- Block 3: For each $\left\{u_{i_{1}}, u_{i_{2}}, u_{i_{3}}\right\}=S_{i} \in S$ a set of $n-m+2$ voters. Three with profiles of the form $\cdots \succ w \succ t \succ u_{i_{j}} \succ S_{i}$, where $j \in\{1,2,3\}$, and all others of the form $\cdots \succ w \succ t \succ S_{i}$;

- Block 4: For each $u_{k} \in U$ a set of $n-m-1$ voters with profiles of the form $\cdots \succ w \succ t \succ u_{k}$;

- Block 5: A set of $n-m-1$ voters with profiles of the form $\cdots \succ w \succ t$

- Block 6: A set of $n-m$ voters with profiles of the form $\cdots \succ t \succ w$.

Let us now show why the existence of an equilibrium profile that solves $\operatorname{Win} N E(w, \mathbf{a})$, where $\mathbf{a}$ is as described above, entails a solution to the $\mathrm{X} 3 \mathrm{C}$ instance. To this end, consider the voters' behaviour in a NE profile $\mathbf{b}$, where $w$ is the winner. 
According to Lemma $1, \operatorname{sc}(w, \mathbf{b})=\operatorname{sc}(w, \mathbf{a})$, yet by our construction $\operatorname{sc}(t, \mathbf{a})=$ $\operatorname{sc}(w, \mathbf{a})+1$. Hence, for $w$ to become a winner in $\mathbf{b}, t$ has to receive at least one additional veto and will also be the threshold candidate (if $t$ is not a runner-up, it means several are no longer vetoing it, but as they are vetoing some non-winner instead, they can revert to being truthful and gain $\epsilon$ utility. Due to its loss to $w$ in tie-breaking, it means $t$ is the threshold candidate). According to Lemma 3 and the truth bias assumption, none of those who vetoed $t$ in a would switch to veto another candidate. In fact, there is only one voter that needs to deviate from its truthful profile and veto $t$.

Consider the voters of Block 2. $(n-m)$ of them prefer $t$ to $w$, and would not veto the former. Yet, if they lie in $\mathbf{b}$, they have to veto a runner up. As a result, none of them can deviate from their truthful profile in equilibrium. On the other hand, the last voter of the block can (and should) deviate, and veto $t$.

Similarly, consider the score of $p_{1}$ in the truthful profile a and compare it to that of $\operatorname{sc}(w, \mathbf{a})$. For $w$ to be the winner of the equilibrium profile $\mathbf{b}, m$ voters from Block 1 need to deviate in the equilibrium and stop vetoing $p_{1}$. These newly vetoed candidates have to be less preferred than $w$ by the deviating voters. For voters of Block 1, this means vetoing a candidate from the set $S$. As a result, there are $m$ candidates $S_{i} \in S$ that are being vetoed by the voters from Block 1 in the equilibrium profile $\mathbf{b}$.

These chosen $S_{i}$ 's, however, need to be runner-up candidates. To achieve that, exactly 3 candidates that veto $S_{i}$ 's in Block 3 must deviate in the equilibrium profile $\mathbf{b}$. These can only be the voters with preference profiles of the form $\cdots \succ w \succ t \succ u_{i_{j}} \succ$ $S_{i}$, where $j \in\{1,2,3\}$.

Since no voter in Block 4 can deviate, those voters from Block 3 that deviate to veto $u_{i_{j}}$ s can only do so consistently with Lemma 3 , if the total number of times that $u_{i_{j}}$ is being vetoed is equal to $(n-m)$. This can happen only if each $u_{i_{j}} \in U$ is vetoed exactly once by voters from Block 3 .

As a result, the sub-set $S_{i}$ 's that are vetoed by voters in Block 1 constitutes a solution to the given $\mathrm{X} 3 \mathrm{C}$ instance. The opposite direction, that is, constructing a NE profile given a solution to the $\mathrm{X} 3 \mathrm{C}$ instance, is trivial.

The results of this subsection show that there are critical properties of the truthful profile a that make the existence of an equilibrium with a given winner a hard problem. However, as we show in the next subsection, combining these properties, namely condition $C 1$ and $C 2$, creates a polynomial-time decidable sub-class.

\subsection{A Polynomially Solvable Subclass}

In the previous subsection, we demonstrated two conditions on a candidate and the truthful profile that, if violated, make WinNE, under truth-biased voters, NP-hard. In this subsection, we complete our treatment on this categorization of profiles by considering the subset where both aforementioned conditions hold. In fact, we provide a constructive proof, via a reduction to a max-flow problem, showing that in this sub-class of truthful profiles, $\operatorname{Win} N E(w, \mathbf{a})$ can be decided in polynomial time.

Theorem 4. Consider a candidate $w \in C$ and a truthful profile a for which conditions $C 1$ and $C 2$ hold. Then $W i n N E(w, \mathbf{a})$, i.e., the existence of a $N E$ profile $\mathbf{b}^{N E}$, where $\mathcal{F}\left(\mathbf{b}^{N E}\right)=w$, is decidable in polynomial time. 
The statement of the above theorem is tight, given Theorems 1 and 3. Namely, should either one of the conditions $C 1$ or $C 2$ be violated, determining the existence of a NE with $w$ as the winner becomes NP-hard. The conditions $\mathrm{C} 1$ and $\mathrm{C} 2$ ensure that we can focus on a particular threshold candidate (namely candidate $t$ ) for constructing a Nash equilibrium profile. While $C 1$ ensures some manipulation will be necessary (and the preference order ensures it will be a threshold), it is $C 2$ that ensures that $t$ can be a valid threshold candidate, since without it, as Observation 1 noted, this is not possible.

Proof. Consider an instance of the problem specified by a potential winner $w \in C$ and the real profile a. Let $t$ also be the candidate right next to $w$, as specified by conditions $\mathrm{C} 1$ and $\mathrm{C} 2$. The proof is based on a polynomial reduction to the max-flow problem in a graph. We will construct a graph (and later correct the flow) in such a way that the set of flow-saturated edges will indicate the feasibility of obtaining a Nash equilibrium. Furthermore, positive flow at certain nodes in the graph will indicate a switch in the voters' equilibrium ballots from their truthful profile.

Given a truthful voting profile a, we will construct the graph as follows. Vertices will be associated with each candidate and each voter; we also add a source and a sink node. The set of graph vertices will therefore be $\{$ source, sink $\} \cup C \cup V$.

The set of edges, $E$, in the graph will consist of three subsets.

- Potential deviators. Edges that link voters and potentially vetoed candidates.

For a voter $i$ where the last candidate in its real preference order is some $r \in C$, i.e., the preference order is in the form $\ldots \succ w \succ c_{1} \succ \ldots \succ c_{l} \succ r$, add the following directed edges with unit flow capacity: $\left(r, v_{i}\right),\left(v_{i}, c_{1}\right), \ldots,\left(v_{i}, c_{l}\right)$.

The resulting palm-leaf sub-structure is depicted in Figure 1. It essentially captures the ability of the voter to change its veto in a manner that will benefit $w$ without deteriorating the voter's utility (note, of course, that there are multiple such sub-graphs in the graph, and vertices may be part of several such structures).

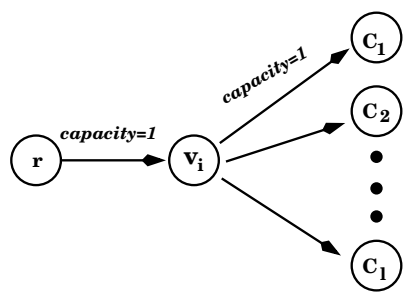

Fig. 1. Polytime special veto subclass. Palm sub-structure for Theorem 4.

- Sustainable deviations. Edges from the source node. These edges and capacities reflect the number of additional points a candidate may absorb until it becomes a runnerup candidate w.r.t. $w$. Hence (recall that $t$ is the candidate next to $w$ in the tie-breaking order, as specified by conditions $\mathrm{C} 1$ and $\mathrm{C} 2$ ):

For each candidate $c$ so that $t \succ c$ in tie-breaking and $\operatorname{sc}(w, \mathbf{a})-\operatorname{sc}(c, \mathbf{a})>0$, a directed edge $($ source, $c)$ is added with capacity $\operatorname{sc}(w, \mathbf{a})-\operatorname{sc}(c, \mathbf{a})$.

For each candidate $c$ so that $c \succ w$ in tie-breaking and $\operatorname{sc}(w, \mathbf{a})-\operatorname{sc}(c, \mathbf{a})>1$, a directed edge $(\operatorname{source}, c)$ is added with capacity $\operatorname{sc}(w,, a)-\operatorname{sc}(c, \mathbf{a})-1$. 
- Necessary deviations. Edges to the sink node. These edges and capacities reflect the number of additional veto votes a candidate needs to sustain to make its score less than that of $w$. Otherwise, $w$ would not be able to become the winner.

For each candidate $c$ so that $t \succ c$ in tie-breaking and $\operatorname{sc}(w, \mathbf{a})-\operatorname{sc}(c, \mathbf{a})<0$, a directed edge $(c, \operatorname{sink})$ is added with capacity $\operatorname{sc}(c, \mathbf{a})-\operatorname{sc}(w, \mathbf{a})$.

For each candidate $c$ so that $c \succ w$ in tie-breaking and $\operatorname{sc}(w, \mathbf{a})-\operatorname{sc}(c, \mathbf{a})<1$, a directed edge $(c, \sin k)$ is added with capacity $1-(\operatorname{sc}(w, \mathbf{a})-\operatorname{sc}(c, \mathbf{a}))$.

Given Corollary 1, the non-truthful votes at an equilibrium profile come from voters that were not vetoing $w$ in $\mathbf{a}$, and they now lie by vetoing some candidate other than their truthful vetoed candidate, which is less-preferred than $w$. From the construction of the graph, it is easy to see that if the maximal flow through the above graph is less than the sum of all incoming capacities to the sink node, then there can be no equilibrium profile that makes $w$ a winner. To see this, observe that only candidate vertices connect directly to the sink, and these are precisely candidates that have higher scores than $w$. Total capacity of all these edges equals the number of voters that necessarily have to change their vote. Furthermore, the flow has to go through voter vertices, connected to candidates that are less-preferred to $w$ (and hence indicate a switch from the truthful vote to a non-truthful one). Finally, if all edges to the sink are saturated in a maximum flow, we will show that a Nash equilibrium profile $\mathbf{b}^{N E}$, with $\mathcal{F}\left(\mathbf{b}^{N E}\right)=w$, can be recovered from the flow. In what follows we will demonstrate this formally.

Let $f: E \rightarrow \mathcal{R}$ be a maximal acyclic, integer flow through the constructed graph. Such a flow can be obtained in time polynomial in the number of voters and candidates. Furthermore, all edges from a candidate node to a voter node that have positive flow on them will be saturated (as their capacity is 1).

We will now modify the flow, while maintaining its total capacity, to maximize the flow through the source outgoing edges, and minimize the flow through voter nodes. Since we will later associate a flow through a voter node with the voter deviating from the truthful vote, minimizing the flow through voter nodes will reflect and ensure that the voting profile recovered from it will be truth biased (i.e., no unnecessary lying takes place, otherwise some voter would have an incentive to switch back to the truthful vote).

Let $D=\{c \mid \exists e=($ source,$c) \in E\}$ be the set of all nodes directly connected to the source. Notice that $D$ is a subset of candidate nodes. Let $q \in D$ be a node for which: $i)$ there is a voter $v$ so that $(v, q) \in E ; i i) f((v, q))>0$; and iii) the edge (source, $q$ ) is not saturated. We will repeat the following flow modification until no such $q$ exists.

Consider a flow path to $q$ through voter nodes. In particular, let $\pi=($ source $=$ $\left.n_{0}, n_{1}, \ldots, n_{l}=q\right)$ be an acyclic path from the source to $q$, so that $e_{k}=\left(n_{k-1}, n_{k}\right) \in$ $E$ for all $k \in[l]$ and $f\left(e_{k}\right)>0$. Notice that since $f$ is an integer flow and all edges between candidate nodes and voter nodes have unit capacity, all the edges of the path have a unit flow apart from the initial edge from the source to $n_{1}$. We will modify the flow $f$ and construct an augmented flow $\widehat{f}$ by canceling the flow through $\pi$, and replacing it with an additional unit flow from the source to $q$. More formally, let $\widehat{f}=f$. We then set $\widehat{f}\left(e_{k}\right)=0$ for all $k \in[1: l-1], \widehat{f}\left(\left(\right.\right.$ source,$\left.\left.n_{1}\right)\right)=f\left(\left(\right.\right.$ source,$\left.\left.n_{1}\right)\right)-1$ and $\widehat{f}(($ source,$q))=f(($ source, $q))+1$. We then repeat the modification procedure, if 
necessary, for $\widehat{f}$. Notice that the flow modification procedure does not change the total flow from the source to the sink node.

Assume now that the flow is such that for all nodes $q \in D$, either the edge (source, $q$ ) is saturated, or $q$ has no positive incoming flow from the voter nodes. Then for every voter $v_{i} \in V$, if there is an edge $\left(v_{i}, c_{j}\right)$ for some $c_{j} \in C$, so that $f\left(v_{i}, c_{j}\right)>0$, i.e., saturated, we let $v_{i}$ change its vote to veto $c_{j}$. Otherwise, $v_{i}$ votes truthfully. Let $\mathbf{b}^{N E}$ be the resulting strategy profile. It is easy to see that $\mathbf{b}^{N E}$ is indeed an equilibrium.

Notice that the equilibrium profile, $\mathbf{b}^{N E}$, was constructed in poly-time. Recall, the steps consisted of: a) constructing the graph, which takes time polynomial in the number of candidates, $m$, and voters, $n$; b) finding a maximal acyclic integer flow (poly-time algorithms exist, and any one is suitable); c) a set of flow modifications. Finding the path $\pi$ necessary for the flow modification takes polynomial time in $m$ and $n$, e.g., by following the flow $f$ back through the saturated edges. Furthermore, the number of repetitions of the flow modification process is polynomial in the number of candidates and voters as well. This is because the flow through any candidate node from voter nodes is bounded from above by the number of voters, and is reduced by one in every modification. As a result, the running time of the whole algorithm is polynomial.

Note that we cannot have an analogous separation for the problem $\exists N E$, since the conditions $\mathrm{C} 1$ and $\mathrm{C} 2$ depend on the winner under consideration. Hence, we can clearly have a polynomial time algorithm for $\exists N E$, if $\mathrm{C} 1$ and $\mathrm{C} 2$ hold for every $w \in$ $C$ (running the algorithm for $\operatorname{WinNE}(w, \mathbf{a})$ for every $w$ ), but we cannot conclude anything if these conditions do not hold across all candidates.

\section{Truth-bias Under k-approval}

The veto rule and the plurality rule are two extreme points of the general family of $k$ approval voting. For $k$-approval with $k \geq 1$, voters "approve" of the first $k$ candidates in their submitted ballot, hence each such candidate receives one point from that voter. In this section, we briefly show that we cannot hope to have analogous results as in Section 3. Clearly the NP-hardness results continue to hold, since veto is included in $k$-approval, but the rest of the properties that we identified in the previous section do not hold for the more general class of $k$-approval rules.

For the analysis, we will adopt the general terminology of Section 3. In more detail, we will use the same definition of a runner-up candidate (as well as the definitions for the sets $\mathbf{R}_{1}, \mathbf{R}_{2}$, and for threshold candidates), as the ones used for the veto case. Additionally, we will denote the set of approved candidates in a profile $a_{i}$ (respectively, $b_{i}$ ) by $\mathcal{A}_{i}$ (respectively, $\mathcal{B}_{i}$ ). Finally we will use the terms "votes in favor" and "votes against" in the sense of the following definition.

Definition 7. Let $\mathbf{a}$ be the truthful profile, and let $\mathbf{b}$ be the submitted profile. A voter $i$ votes in favor of a candidate $c_{j}$, if $c_{j} \notin \mathcal{A}_{i}$ and $c_{j} \in \mathcal{B}_{i}$. Similarly, $i$ votes against $c_{j}$, if $c_{j} \in \mathcal{A}_{i}$ and $c_{j} \notin \mathcal{B}_{i}$.

Lemma 5. Given a Nash equilibrium profile $\mathbf{b}^{N E} \neq \mathbf{a}$, for every non-truhful voter, exactly one, but never both, of the following conditions hold: a) the voter votes in favor of the winner, $b$ ) the voter votes against some $r \in R_{1} \cup R_{2}$. 
The lemma above already shows some differentiation from what holds for veto. Now, note that for veto, it is always case (b) from Lemma 5 that holds, and never case (a). For plurality, on the contrary, it was established in [14] that it is case (a) that holds, and never case (b). For $k$-approval, with arbitrary $k$, it can be either of the two cases.

Next, we establish that a threshold candidate always exists as in the previous section.

Proposition 1. For every equilibrium $\mathbf{b}^{N E} \neq \mathbf{a}$, a threshold candidate always exists.

Unlike the case of veto or plurality, in the $k$-approval case, it is possible that in a non-truthful equilibrium, neither the winner nor the threshold candidate will maintain the same score as in the truthful profile. This is demonstrated in the following examples.

Example 2. Consider the following two profiles using 2-approval, with the tie-breaking order given by the sequence $a \succ b \succ c \succ d \succ e$. The truthful profile (of 7 voters) is:

$$
\begin{aligned}
& \text { - } a \succ b \succ c \succ d \succ e \quad \text { - } 2 \text { voters with preference } d \succ b \succ a \succ c \succ e \\
& \text { - } e \succ d \succ a \succ c \succ b \quad \text { - } 2 \text { voters with preference } a \succ d \succ b \succ c \succ e \\
& \text { - } e \succ c \succ a \succ b \succ d
\end{aligned}
$$

The equilibrium profile changes the last but one voter (i.e., one out of the two identical voters with preference $a \succ d \succ b \succ c \succ e$ ), as well as the other one of that type and the last voter to $a \succ e \succ b \succ c \succ d$ and $e \succ a \succ c \succ b \succ d$

In this example, the score of the winning candidate (more specifically, candidate $a$ ) in the equilibrium profile is higher than in the truthful profile. On the other hand, the score of the threshold candidate in the equilibrium (in this example $d$ ) decreases in the equilibrium compared to the truthful profile score.

Example 3. The score of the threshold candidate does not necessarily decrease at an equilibrium. A further example demonstrates that its score can also become larger in an equilibrium than it is in the truthful profile. Using again 2-approval, and the tie-breaking order $d \succ a \succ b \succ c$, this example consists of the following true preferences:

$$
\begin{array}{rlrl}
a \succ b & \succ c \succ d & & a \succ c \succ b \succ d \\
c \succ d & \succ a \succ b & & d \succ b \succ a \succ c \\
a \succ d \succ b & \succ c
\end{array}
$$

A NE can be constructed by making only one change - the last voter changes to $a \succ b \succ d \succ c$. The threshold candidate score in the equilibrium (here of candidate $b$ ) increases.

Finally, regarding the winner's score in a Nash equilibrium, we prove that it cannot fluctuate and go up or down as the score of the threshold candidate, but instead it is bounded, according to the following proposition.

Proposition 2. Let $w=\mathcal{F}\left(\mathbf{b}^{N E}\right)$ for a $N E \mathbf{b}^{N E} \neq \mathbf{a}$. Then $\operatorname{sc}\left(w, \mathbf{b}^{N E}\right) \geq \operatorname{sc}(w, \mathbf{a})$.

Our results along with a comparison to the plurality rule are summarised in Table 2. One of the main conclusions drawn from Table 2 is the following: the analysis of the plurality rule by Obraztsova et al. [14] established that the score of the threshold candidate in a non-truthful equilibrium remains the same as in the truthful profile. This property was exploited in that work for characterising the set of equilibria. Regarding 
the veto rule, the score of the threshold candidate may change, but the score of the winner remains unchanged among equilibria. This is a property that we exploited in Section 3 in our positive result. In contrast, when it comes to the general family of $k$ approval, the examples in this section show that the score of both the winner and the threshold candidate may change. The threshold candidate in particular is far less constrained, and can either lose or gain points in the equilibrium compared to the truthful profile. Hence, equilibrium profiles are not as well-structured in this case, and therefore we cannot hope to use the ideas from Section 3 or from the plurality analysis of Obraztsova et al. [14] to come up with more positive algorithmic results. Our analysis suggests that one may have to look at special cases of $k$-approval to obtain structural properties that can yield characterizations, or can be exploited algorithmically.

Table 2. Summary of our complexity results and other properties.

\begin{tabular}{|c|c|c|c|c|}
\hline Conditions & Veto & & Plurality & $k$-approval \\
\hline & 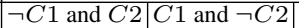 & $C 1$ and $C 2$ & & \\
\hline $\operatorname{WinNE}(w, \mathbf{a})$ & NP-hard & $\mathrm{P}$ & NP-hard & NP-hard \\
\hline Winner score may grow in NE & \multicolumn{2}{|l|}{ No } & Yes & Yes \\
\hline Winner score may drop in NE & \multicolumn{2}{|l|}{ No } & No & No \\
\hline Runner-up score may grow in NE & \multicolumn{2}{|l|}{ Yes } & No & Yes \\
\hline Runner-up score may drop in NE & \multicolumn{2}{|l|}{ Yes } & No & Yes \\
\hline
\end{tabular}

\section{Discussion and Future Work}

In this paper we initiated the investigation of truth-biased voters in voting systems that do not use plurality. We focused on the spectrum of voting rules ranging from plurality to veto, encompassing all scoring rules that allow only two values. In these cases, we have seen that plurality, veto, and $k$-approval have their own distinct properties. For example, both plurality and $k$-approval share the property that the score of an equilibrium winner might be higher than the truthful score of that candidate (which cannot happen in veto). However, in $k$-approval, unlike plurality, equilibria exist where runner-up candidates also change their scores.

While the problems we studied are NP-complete for both veto and $k$-approval, we showed a tight subset of cases for veto where there is a polynomial time algorithm for knowing (and finding, as the algorithm is constructive) if there is a Nash equilibrium with a winner of our choice.

There are several further research areas to pursue. First, we can combine this research with other voting approaches. For example, while there has been research on iterative voting with truth-bias, it has only focused on plurality.

A different approach remains strictly within the framework of truth-bias, and tries to further enhance our understanding of truth-biased voters, and expand the research of it to more voting rules (most interesting, to non-scoring rules, such as maximin), allowing us to further understand the effects of truth-bias. However, this approach presents unique challenges, as while truth-bias in binary scoring effectively eliminates the most egregious of nonsensical equilibria, expanding it to other voting rules requires a wider net, in a sense, to eliminate such equilibria. 


\section{Acknowledgements}

This research was supported in part by Israel Science Foundation grant \#1227/12, Israel Ministry of Science and Technology grant \#3-6797, and by Microsoft Research through its PhD Scholarship Programme. It has also been supported by the EU (European Social Fund) and Greek national funds through the Operational Program "Education and Lifelong Learning" of the National Strategic Reference Framework (NSRF) - Research Funding Program: THALES. The work of S. Obraztsova was partially supported by ERC grant \#337122 under the EU FP7/2007-2013 and RFFI grant 14-01-00156-a.

\section{References}

1. Branzei, S., Caragiannis, I., Morgenstern, J., Procaccia, A.D.: How bad is selfish voting? In: AAAI (2013)

2. Desmedt, Y., Elkind, E.: Equilibria of plurality voting with abstentions. In: ACM EC. pp. 347-356 (2010)

3. Dutta, B., Laslier, J.F.: Costless honesty in voting (2010), in 10th International Meeting of the Society for Social Choice and Welfare, Moscow

4. Dutta, B., Sen, A.: Nash implementation with partially honest individuals. Games and Economic Bahavior 74(1), 154-169 (2012)

5. Farquharson, R.: Theory of Voting. Yale University Press (1969)

6. Garey, M.R., Johnson, D.S.: Computers and Intractability: A Guide to the Theory of NPCompleteness. Series of Books in the Mathematical Sciences, W. H. Freeman (1979)

7. Gibbard, A.: Manipulation of voting schemes. Econometrica 41(4), 587-602 (1973)

8. Laffont, J.J.: Incentives and the allocation of public goods. In: Handbook of Public Economics, vol. 2, chap. 10, pp. 537-569. Elsevier (1987)

9. Laslier, J.F., Weibull, J.W.: A strategy-proof condorcet jury theorem. Scandinavian Journal of Economics (2012)

10. Lev, O., Rosenschein, J.S.: Convergence of iterative voting. In: AAMAS. pp. 611-618 (2012)

11. Meir, R., Polukarov, M., Rosenschein, J.S., Jennings, N.R.: Convergence to equilibria of plurality voting. In: AAAI. pp. 823-828 (2010)

12. Messner, M., Polborn, M.K.: Miscounts, duverger's law and duverger's hypothesis. Tech. Rep. 380, Innocenzo Gasparini Institute for Economic Research, Bocconi University (2011)

13. Myerson, R.B., Weber, R.J.: A theory of voting equilibria. The American Political Science Review 87(1), 102-114 (1993)

14. Obraztsova, S., Markakis, E., Thompson, D.R.M.: Plurality voting with truth-biased agents. In: SAGT. pp. 26-37 (2013)

15. Rabinovich, Z., Obraztsova, S., Lev, O., Markakis, E., Rosenschein, J.S.: Analysis of equilibria in iterative voting schemes. In: Proceedings of the 29th AAAI Conference on Artificial Intelligence (AAAI). pp. 1007-1013. Austin, Texas (January 2015)

16. Satterthwaite, M.A.: Strategy-proofness and Arrow's conditions: Existence and correspondence theorems for voting procedures and social welfare functions. Journal of Economic Theory 10(2), 187-217 (1975)

17. Thompson, D.R.M., Lev, O., Leyton-Brown, K., Rosenschein, J.S.: Empirical aspects of plurality election equilibria. In: AAMAS (2013)

18. Xia, L., Conitzer, V.: Stackelberg voting games: Computational aspects and paradoxes. In: AAAI. pp. 805-810 (2010) 